\title{
QUAN ĐIỂM CỦA SINH VIÊN VỀ THỤ̉C TRẠNG CHƯƠNG TRİNH ĐÀO TẠO TIẾNG ANH NHIẸM VỤ CHIẾN LỰ̛C TẠI ĐẠI HỌC QUỐC GIA HÀ NỘI
}

\author{
Huỳnh Anh Tuấn ${ }^{1}$ *, Đỗ Thị Anh Thư² \\ ${ }^{1}$ Khoa Sau đại học, Truờng Đại học Ngoại ngũu, ĐHQGHN, Phạm Văn Đồng, \\ Cầu Giấy, Hà Nội, Việt Nam \\ ${ }^{2}$ Khoa Ngoại ngũu, Trưòng Đại hoc Hàng hải Việt Nam, 484 Lạch Tray, \\ Ngô Quyền, Hải Phòng, Việt Nam \\ NCS Khóa QHF2016, Trưòng Đại học Ngoại ngũ, ĐHQGHN \\ Nhận bài ngày 15 tháng 1 năm 2017
}

Chỉnh sửa ngày 20 tháng 3 năm 2017; Chấp nhận đăng ngày 22 tháng 3 năm 2017

Tóm tắt: : Bài báo này báo cáo một phần kết quả của một đề tài nghiên cứu do Đại học Quốc gia Hà Nội (ĐHQGHN) tài trợ (Mã số: QG.13.13). Bài báo phản ánh, phân tích hiện trạng đào tạo chương trình giảng dạy tiếng Anh nhiệm vụ chiến lược (NVCL) dựa trên kết quả điều tra, khảo sát ý kiến, quan điểm của sinh viên tham gia chương trình về các hoạt động dạy và học của chương trình nhằm đưa ra những kiến nghị, đề xuất nâng cao chất lượng đào tạo của chương trình và chất lượng đầu ra cho sinh viên. Kết quả điều tra khảo sát cho thấy dù còn một số vấn đề cần được năng cao, cải tiến, hầu hết sinh viên nhận định đây là một chương trình đào tạo chất lượng, có hiệu quả, phù hợp với đường hướng giáo dục dựa vào chuẩn đầu ra.

Tù khóa: chương trình tiếng Anh nhiệm vụ chiến lược, đào tạo tiếng Anh tập trung tăng cường, đường hướng giáo dục dựa vào chuẩn đầu ra, năng lực tiếng Anh

\section{Chương trình tiếng Anh NVCL của ĐHQGHN}

\subsection{Giới thiệu chung về Chưong trình tiếng Anh NVCL của ĐHQGHN}

Từ năm học 2010-2011, Trường ĐHNNĐHQGHN được ĐHQGHN giao nhiệm vụ giảng dạy tiếng Anh cho sinh viên năm thứ nhất hệ đào tạo chuẩn quốc tế (International Standard Programme, gọi tắt là ISP, tên chương trình bằng tiếng Việt là Chương trình tiếng Anh NVCL). Các đối tượng sinh viên này đang theo học 19 ngành học khác nhau của các trường đại học thành viên của

${ }^{1}$ Tác giả liên hệ. ĐT.: 84-902229101

Email: huynhanhtuan@vnu.edu.vn
ĐHQGHN. Ngoài việc học tập trên lớp, sinh viên còn được hướng dẫn, hỗ trợ ngoài giờ và tham gia rất nhiều các hoạt động ngoại khóa để nâng cao trình độ tiếng Anh. Sau một năm đào tạo tập trung tại Trường ĐHNN-ĐHQGHN sinh viên phải đạt được trình độ tiếng Anh tối thiểu là 7.0 IELTS, hiện là bậc $5 / 6(\mathrm{C} 1)$ theo Khung năng lực ngoại ngữ dùng cho Việt Nam (KNLNNVN), đủ năng lực tiếng Anh để theo học các môn chuyên ngành bằng tiếng Anh khi trở về trường.

Sinh viên NVCL phải tham gia vào kỳ thi phân loại để xác định trình độ đầu vào tiếng Anh phù hợp, phục vụ cho việc xếp lớp. Dựa vào trình độ đầu vào tiếng $\mathrm{Anh}$, sinh viên $\mathrm{NVCL}$ 
được xếp vào 2 nhóm trình độ khác nhau, chia thành khối sáng và chiều. Mỗi nhóm trình độ được áp dụng chương trình giảng dạy khác nhau, phù hợp với trình độ của các em. Các nhóm có trình độ yếu hơn sẽ được giáo viên giảng dạy tăng cường hỗ trợ trong việc hướng dẫn và cung cấp giáo trình tự học. Các em còn được sinh viên hệ chất lượng cao (CLC) của Trường ĐHNN hỗ trợ hướng dẫn ngoài giờ. Việc chia sinh viên thành hai nhóm trình độ khác nhau chỉ được duy trì ở hai học kỳ đầu tiên A1-A2. Đến học kỳ B1, các lớp đều học chung một chương trình và tập trung phát triển các kỹ năng ngôn ngữ Nghe, Nói, Đọc, Viết.

Một năm học của SV NVCL được chia thành 05 học kỳ từ A1-C1. Mỗi học kỳ kéo dài 06 tuần. Mỗi tuần sinh viên học 20 tiết tiếng Anh tập trung. Sau mỗi học kỳ đều có một bài kiểm tra tiếng Anh ở cả 4 kỹ năng để đánh giá sự tiến bộ và năng lực tiếng của sinh viên. Mỗi lớp NVCL đều có 01 giáo viên chủ nhiệm để theo dõi hoạt động học tập của sinh viên. Ngoài chương trình học tập trên lớp, $\mathrm{SV}$ NVCL còn được tham gia rất nhiều các hoạt động ngoại khóa bằng tiếng Anh, giúp các em tự tin trong giao tiếp và không ngừng tạo động cơ trong học tập như tham gia câu lạc bộ tiếng Anh, xem phim tiếng Anh, học thêm các khóa học ở các trung tâm, luyện phát âm theo các video trên mạng...

Sinh viên được học qua rất nhiều nguồn học liệu như giáo trình học trên lớp, giáo trình bổ trợ, giáo trình trực tuyến, trên thư viện, trên mạng. Ngoài ra, giáo viên cũng hướng dẫn các em tìm thêm các nguồn tài liệu tham khảo khác và tự chuẩn bị các nguồn học liệu cho mình. Sau 05 năm triển khai, chương trình giảng dạy TA NVCL đã có những đóng góp đáng kể, giúp nâng cao chất lượng tiếng Anh đầu ra của sinh viên.
Năm học 2011-2012, số sinh viên NVCL QH2011 đạt chuẩn đầu ra C1 (tương đương 7.0 IELTS) là 45\%; số sinh viên đạt chuẩn đẩu ra năm thứ nhất (tương đương 6.5 IELTS với mức điểm này sinh viên có đủ năng lực để theo học các môn chuyên ngành bằng tiếng Anh) là 75.5\%. Năm học 2012-2013, số sinh viên NVCL QH2012 đạt chuẩn đầu ra C1 là 50,7 \% ; số sinh viên đạt chuẩn đẩu ra năm thứ nhất là 86,1\%. Năm học 2013-2014, số sinh viên NVCL QH2013 đạt chuẩn đầu ra C1 là $76.1 \%$; số sinh viên đạt chuẩn đẩu ra năm thứ nhất là $84,8 \%$.

1.2. Chuoong trình đào tạo tiếng Anh tập trung tăng cương (tuoong đương chuoong trình NVCL)

Theo Davies (2006), mô hình đào tạo tập trung tăng cường (intensive teaching models/ ITM, intensive modes of delivery/IMD) còn gọi là khoá học tăng tốc (accelarated), thời khối (block format/block teaching), rút gọn (time-shortened), hay nén (compressed).

Các khoá đào tạo theo thời khối (block format/block teaching) là hình thức đào tạo theo "thời khoá biểu hàng ngày được tổ chức thành những thời khối lớn hơn 60 phút cho phép sự linh hoạt, phong phú của các hoạt độnh giảng dạy" (Cawelti, 1994). Hình thức này đã được thử nghiệm ở bậc đại học và đã đạt được những thành công nhất định với mô hình 2 thời khối 80 phút/tuần so với mô hình 3 thời khối 50 phút/tuần thông thường (Gaubatz, 2003). Hình thức đào tạo tăng tốc hay tập trung tăng cường (accelerated/intensive teaching) là hình thức đào tạo với thời gian tương tác giữa người học và người dạy ít hơn thường lệ, khoảng 25 giờ trên lớp trong 5 tuần hoặc 8 tuần so với 45 giờ trên lớp trong 16 tuần (Scott \& Conrad, 1992; Wlodkowski, 2003). Hình thức đào tạo 
này bao hàm các dạng thức nén trong giảng dạy (compressed teaching formats) được triển khai trong các lớp học ngoài giờ vào cuối tuần hoặc các buổi tối trong tuần. Các khoá học này phù hợp với bậc đại học hơn bậc phổ thông.

Davies (2006) cho rằng hầu hết các nghiên cứu so sánh hình thức đào tạo tăng cường tập trung và hình thức đào tạo truyền thống cho thấy hoặc không có khác biệt về kết quả học tập giữa 2 hình thức hoặc có sự tiến bộ về kết quả học tập đối với hình thức tăng cường tập trung tuỳ thuộc vào độ nén, độ tăng tốc của từng chương trình, từng môn học và từng cơ sở đào tạo.

Ngoài hình thức đào tạo tăng cường tập trung nêu trên còn một số hình thức đào tạo tăng cường tập trung phổ biến sau (Finger \& Penney, 2001): Khoá học 1 tuần: kéo dài từ 5 đến 6 ngày liên tục từ 8 h30 sáng đến 4 h30 chiều (Clark \& Clark, 2000; Grant, 2001); Khoá học 2-3 tuần (Petrowsky, 1996; van Scyoc \& Gleason, 1993); Khoá học cuối tuần: được tổ chức vào các cuối tuần thứ 3 , thứ 6 và thứ 9 của 1 học kỳ; Khoá học cuối tuần và buổi tối: kết hợp giữa hình thức khoá học cuối tuần và một số buổi tối trong tuần; Khoá học phi chính thống: được thực hiện 3 giờ/ngày trong 18 ngày (Gose, 1995); 3 giờ/tuần (Henebry, 1997); 4 giờ/tuần trong 5-10 tuần (Jonas, Weimer \& Herzer, 2004). Davies (2006) nhận định hình thức đào tạo tập trung tăng cường hoàn toàn phù hợp với xu thế giáo dục hướng chuẩn đầu ra (Berlach, 2004; Evans, 1994; Killen, 2000; Kohn, 1993; Spady, 1994).

\section{Phương pháp nghiên cứu}

\subsection{Câu hỏi nghiên cứu}

Nghiên cứu được tiến hành với mục đích tìm ra câu trả lời cho câu hỏi: "Sinh viên tham gia chương trình NVCL có những đánh giá, nhận xét gì về các hoạt động liên quan đến việc dạy và học của chương trình?” Nói cách khác, nghiên cứu đặt ra mục tiêu tìm hiểu quan điểm của SV NVCL về các yếu tố có liên quan đến chất lượng đào tạo của chương trình. Thuật ngữ quan điểm trong bài báo được hiểu là các ý kiến nhận xét, đánh giá, tuyên bố mang tính chủ quan, không mang tính kết luận về một vấn đề, một nhận định nào đó. Theo Damer (2008), quan điểm có những đặc tính sau:

- Quan điểm thường mang tính cá nhân

- Các quan điểm khác nhau có mức độ được chấp nhận khác nhau. Vấn đề quan trọng đối với một quan điểm không phải là một cá nhân có quyền hay không có quyền nêu ra một quan điểm mà là quan điểm đó có được chấp nhận hay không. Những quan điểm không dựa trên các căn cứ khoa học hoặc không được kiểm chứng bởi thực tế thường nhận được mức độ chấp nhận thấp hơn những quan điểm có căn cứ.

- Quan điểm cá nhân có thể thay đổi nếu chủ thể đưa ra quan điểm bị thuyết phục bởi một lập luận có căn cứ.

- Các quan điểm khác nhau thường xung đột lẫn nhau và sẽ có những quan điểm bị coi là sai lầm dựa trên một số tiêu chí nào đó.

\subsection{Phuơng pháp nghiên cúu}

Phương pháp nghiên cứu được áp dụng là nghiên cứu khảo sát. Công cụ khảo sát là bảng câu hỏi. Nội dung khảo sát tập trung vào 07 mục sau:

- Công tác giáo viên chủ nhiệm (GVCN)

- Hoạt động kiểm tra, đánh giá (KTĐG)

- Chương trình giảng dạy tiếng Anh NVCL

- Đội ngũ giáo viên (GV) giảng dạy 
- Hoạt động ngoại khóa của sinh viên (SV)

- Chiến lược và phương pháp học tập của $\mathrm{SV}$

- Sự hỗ trợ của Nhà trường.

Việc khảo sát tập trung vào 02 khía cạnh sau:

- Quan điểm của $S V$ về tính cần thiết, mức độ hợp lí của các vấn đề nêu trên

- Quan điểm của SV về mức độ áp dụng của các hoạt động nêu trên của GV trên thực tế.

Lí do của việc tập trung khảo sát quan điểm, ý kiến của sinh viên về các vấn đề trên vì theo chúng tôi đây là những vấn đề trực tiếp tác động đến động cơ, hứng thú học tập, kết quả học tập của sinh viên và chất lượng đào tạo của chương trình. Đây cũng là những vấn đề mà sinh viên có thể nhận thức được và có thể đưa ra những thông tin đáng tin cậy cho việc khảo sát. Các vấn đề khác mà theo chúng tôi không hoàn toàn thuộc phạm vi nhận thức của sinh viên được khảo sát theo quan điểm, ý kiến của giáo viên trực tiếp tham gia giảng dạy chương trình và không thuộc phạm vi trình bày của bài báo này.

Các câu hỏi ở dạng đóng hoặc mở. Các câu hỏi đóng nhằm khảo sát sự đồng thuận của SV đối với những nhận định do nhóm nghiên cứu đưa ra về tính cần thiết, tính hợp lí, mức độ phù hợp, mức độ tác động của các yếu tố trong hoạt động dạy và học của chương trình. Các câu hỏi mở nhằm thu thập ý kiến, quan điểm của $\mathrm{SV}$ về những yếu tố nhóm nghiên cứu có thể chưa đề cập đến trong bảng câu hỏi như các hoạt động cụ thể cần thiết của GVCN, các dạng thức KTĐG, các phương pháp giảng dạy được GV áp dụng trong chương trình, các hoạt động ngoại khóa $\mathrm{SV}$ đã từng tham gia hoặc SV cho là cần thiết, các phương pháp, chiến lược học tập SV đã áp dụng, các hoạt động hỗ trợ của Nhà trường.

Khi thiết lập các câu hỏi trong bảng câu hỏi, nhóm nghiên cứu tuân thủ các nguyên tắc sau:

- Các câu hỏi phải trả lời được và phải rành mạch, rõ ràng (Mackey \& Gass, 2005:96).

- Mỗi câu hỏi chỉ chứa một ý (Nunan, 1992; Johnson, 1992).

- Không tiết lộ thái độ của người nghiên cứu thông qua các câu hỏi dẫn dắt (Nunan, 1992).

- Câu hỏi phải được thử nghiệm trước khi sử dụng chính thức (Mackey \& Gass, 2005; Johnson, 1992).

\section{3. Đối tượng tham gia trả lời khảo sát}

Tham gia trả lời câu hỏi khảo sát là 99 SV NVCL được lựa chọn ngẫu nhiên từ những SV đã và đang theo học chương trình. Đây là những $\mathrm{SV}$ đến từ những trường đại học thành viên của ĐHQGHN. Xấp xỉ $50 \% \mathrm{SV}$ đang học tại trường ĐH KHTN (48\%). Gần 1/3 đang học tại ĐH Công nghệ (28\%). Số còn lại đang học tại ĐH KHXH\&NV (12\%) và ĐH Kinh tế (11\%). Trên 50\% đã học tiếng Anh từ 8-12 năm (54\%). Trên 1/4 đã học tiếng Anh từ 4-7 năm. Số sinh viên đã học trên 12 năm và dưới 4 năm chiếm tỉ lệ tương đối nhỏ lần lượt là $8 \%$ và $11 \%$. Đa số đạt trình độ từ $\mathrm{B} 1$ trở lên ( $88 \%$ ), trong đó tỉ lệ sinh viên đạt trình độ $\mathrm{C} 1$ chiếm 1/3 (33\%). Trên 50\% đạt trình độ B1 (55\%). Tỉ lệ đạt trình độ dưới B1 là $9 \%$ (A1: 1\%; A2; $8 \%)$. Không có sinh viên ở trình độ $\mathrm{B} 2$ và $\mathrm{C} 2$.

\subsection{Phuoong pháp phân tích dũ liệu}

Nghiên cứu kết hợp giữa phương pháp phân tích dữ liệu định lượng và định tính. Phương pháp phân tích định lượng nhằm tìm 
ra tỉ lệ phần trăm SV đồng ý hay không đồng ý với những nhận định nhóm nghiên cứu đưa ra về chương trình. Báo cáo kết quả phân tích tập trung vào những tỉ lệ cao nhất hoặc thấp nhất nhằm đưa ra những nhận định khái quát về quan điểm của $\mathrm{SV}$. Phân tích định tính dựa vào câu trả lời cho những câu hỏi mở của bảng khảo sát để tổng hợp ý kiến của sinh viên theo những chủ đề tìm thấy.

\section{Kết quả điều tra, khảo sát ý kiến của sinh viên về thực trạng chương trình đào tạo tiếng Anh NVCL}

\section{1. Ý kiến, quan điểm của SV về công tác giáo} viên chủ nhiệm (GVCN)

Điều tra $y$ kiến, quan điểm của $S V$ về công tác GVCN bao gồm các nội dung, yêu cầu sau về các hoạt động của công tác GVCN:

- Liên lạc với phụ huynh SV khi cần thiết

- Thông báo kết quả từng kỳ thi đánh giá năng lực cho phụ huynh

- Tổ chức các hoạt động ngoại khóa nhằm mục đích hỗ trợ học tập tiếng Anh

- Tổ chức các hoạt động ngoại khóa nhằm mục đích nâng cao kỹ năng mềm cho $\mathrm{SV}$

- Xây dựng chương trình cố vấn học tập cho SV

- Thông báo về hoạt động của GVCN vào tuần đầu tiên của năm học

- Tiếp SV theo lịch cố định 1 buổi/tuần

- Trực tiếp tham gia giảng dạy lớp chủ nhiệm

- C Cố vấn cho từng SV xây dựng chiến lược học tập cho khóa học

- Tư vấn, định hướng cho $\mathrm{SV}$ về lối sống và quan điểm sống

- Tư vấn, định hướng cho SV về việc bố trí, sắp xếp thời gian biểu cho các hoạt động trong khóa học.
3.1.1. Quan điểm của sinh viên về múc độ cần thiết của các hoạt động liên quan đến công tác GVCN

Theo kết quả khảo sát, các hoạt động của GVCN được đa số sinh viên (từ $90 \%$ trở lên) cho là cần thiết bao gồm việc tổ chức các hoạt động ngoại khóa nhằm mục đích hỗ trợ học tập tiếng Anh và nâng cao kỹ năng mềm cho $\mathrm{SV}$, xây dựng chương trình cố vấn học tập cho sinh viên, và cố vấn cho từng $\mathrm{SV}$ xây dựng chiến lược học tập cho khóa học theo lịch một buổi một tuần. Trên $75 \%$ ủng hộ việc GVCN trực tiếp tham gia giảng dạy lớp chủ nhiệm, các hoạt động tư vấn, định hướng cho $\mathrm{SV}$ về việc bố trí, sắp xếp thời gian biểu cho các hoạt động trong khóa học, tư vấn, định hướng cho $\mathrm{SV}$ về lối sống và quan điểm sống và thông báo về hoạt động của GVCN vào tuần đầu tiên của năm học. Đa số $\mathrm{SV}$ cho rằng việc thông báo kết quả từng kỳ thi đánh giá năng lực cho phụ huynh và liên lạc với phụ huynh SV là không cần thiết. Các tỉ lệ ủng hộ cho các hoạt động này lần lượt là $23 \%$ và $37 \%$. Tiếp SV theo lịch cố định 1 buổi/tuần là hoạt động có tỉ lệ ủng hộ và không ủng hộ gần như ngang bằng nhau với 52\% SV được hỏi ủng hộ hoạt động này.

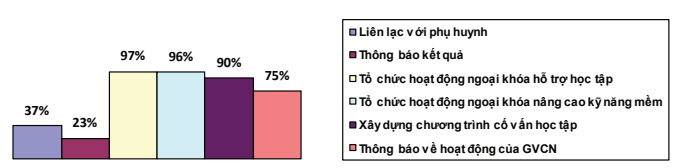

Hình $1 \mathrm{a}$. Tỉ lệ SV cho rằng các hoạt động liên quan đến công tác GVCN nêu ra là cần thiết

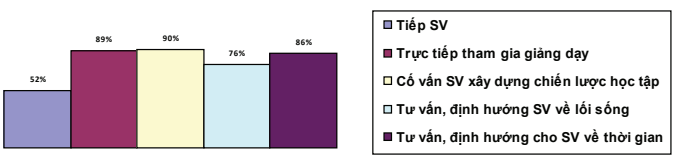

Hình $1 b$. Tỉ lệ $\mathrm{SV}$ cho rằng các hoạt động liên quan đến công tác GVCN nêu ra là cần thiết 
Theo phân tích của chúng tôi, hầu hết các hoạt động có liên quan đến công tác GVCN đều được $\mathrm{SV}$ ủng hộ vì những hoạt động này tích cực hỗ trợ quá trình thụ đắc năng lực tiếng Anh của họ, làm tăng động cơ, hứng thú học tập của người học, tạo môi trường thuận lợi cho người học cải thiện kỹ năng sử dụng tiếng Anh của mình. Tuy nhiên những hoạt động có tác động tiêu cực đến tâm lí của $\mathrm{SV}$ nói chung và thể diện của SV nói riêng như liên lạc với phụ huynh hay thông báo kết quả học tập của SV cho phụ huynh nên được cân nhắc kỹ và trao đổi với $\mathrm{SV}$ để có được sự đồng thuận của họ trước khi thực hiện.

3.1.2. Các hoạt động đã được giáo viên chủ nhiệm triển khai cho chuoong trình tiếng Anh NVCL

Nhìn chung tỉ lệ các hoạt động đã được GVCN áp dụng thấp hơn tỉ lệ được SV cho là cần thiết, dao động từ $52 \%$ đến $76 \%$, ngoại trừ trường $\mathrm{GVCN}$ trực tiếp tham gia giảng dạy lớp chủ nhiệm (89\%). Điều đáng khích lệ là trên $50 \%$ GV đã áp dụng các hoạt động được cho là cần thiết đối với công tác GVCN. Hoạt động được trên $50 \% \mathrm{SV}$ cho là cần thiết, tuy nhiên chỉ dưới 1/3 (29\%) GV đã áp dụng là hoạt động tiếp SV theo lịch cố định 1 buổi/tuần.
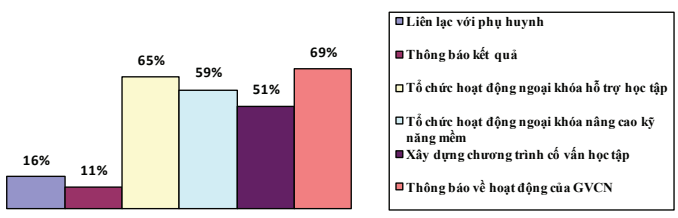

Hình 2a. Các hoạt động đã được GVCN triển khai cho chương trình tiếng Anh NVCL

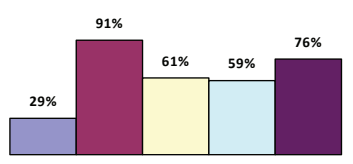

$\square$ Tiếp SV

๑ Trực tiếp tham giagiảng dạy

口Cố vấn SV xây dựng ch hiến lược học tập

口Tư vấn, định hướng SV về lối sống

- Tư vấn, định huớng cho $\mathrm{SV}$ về thời gian

Hình 2b. Các hoạt động đã được GVCN triển khai cho chương trình tiếng Anh NVCL

\subsubsection{Các hoạt động khác đã được} GVCN áp dụng

Ngoài những hoạt động trên, một số các hoạt động khác đã được GVCN áp dụng theo nhìn nhận của $\mathrm{SV}$ :

Thường xuyên theo dõi, kiểm tra, đánh giá kết quả và tình hình học tập (bao gồm kiểm tra bài tập về nhà và bài tập trên lớp) của $\mathrm{SV}$

- Giao bài tập luyện kỹ năng về nhà vào mỗi tuần rồi nộp lại cho $\mathrm{GV}$

- Hướng dẫn chỉ bảo phương pháp học tập và cách để làm bài thi tốt cho $\mathrm{SV}$

- Tăng cường khả năng làm việc theo nhóm cho SV

- Tổ chức các trò chơi bổ ích liên quan đến bài học, giúp SV học tiếng Anh tốt hơn, giúp giờ học bớt căng thẳng, tạo không khí vừa học vừa chơi, vừa giúp học tập vừa giúp các $\mathrm{SV}$ trong lớp gần nhau hơn

- Gắn bó chặt chẽ với $\mathrm{SV}$, quan tâm đến $\mathrm{SV}$, cổ vũ, làm chỗ dựa tinh thần cho $\mathrm{SV}$

Như vậy có thể thấy GV tham gia chương trình hết sức cố gắng, nhiệt tình trong việc tiến hành những hoạt động hỗ trợ người học, quan tâm đến tâm sinh lí người học với mục đích nâng cao hứng thú học tập của người học, được người học ghi nhận và đánh giá cao.

\section{2. Ý kiến SV về các hoạt động kiểm tra đánh giá (KTĐG) của chuoong trình}

Điều tra ý kiến của SV về các hoạt động kiểm tra đánh giá áp dụng trong chương trình liên quan đến các vấn đề sau: 
- Các loại hình KTĐG được áp dụng trong chương trình NVCL

- Mức độ phù hợp của các loại hình KTĐG đã được GV NVCL áp dụng so với mục tiêu đào tạo chung của chương trình tiếng Anh NVCL

- Tác động của các dạng bài kiểm tra tới chiến lược và ý thức học tiếng Anh của SV

- $\quad$ Độ tin cậy của các dạng bài kiểm tra

\subsubsection{Các loại hình KTĐG được áp dụng} trong chuong trình NVCL

Kết quả điều tra cho thấy đa số GV đã áp dụng các loại hình KTĐG khác nhau trong chương trình NVCL như giao bài tập về nhà (99\%), thuyết trình trên lớp và tập bài viết (porfolios) $(84 \%)$, kiểm tra thường xuyên trên lớp (87\%). Các loại hình kiểm tra ít được áp dụng hơn bao gồm kiểm tra giữa kỳ $(29 \%)$ và bài tập dự án (project-based testing) (15\%). Trao đổi về vấn đề này với một số $\mathrm{GV}$ chúng tôi được biết lí do hình thức kiểm tra giữa kỳ chỉ được một số GV áp dụng là SV sẽ trải qua một bài thi đánh giá năng lực cuối kỳ cách thời gian giữa kỳ 3 tuần, một số GV cho rằng trong bối cảnh này bài thi giữa kỳ là không cần thiết. Đối với hình thức kiểm tra dựa vào bài tập dự án, tỉ lệ thấp của việc áp dụng được $\mathrm{GV}$ giải thích là do mức độ quen thuộc thấp (gián tiếp tác động đến mức độ ưa thích và mức độ áp dụng) của cả $G V$ và $S V$ đối với hình thức kiểm tra này.

3.2.2. Mức độ phù hợ của các loại hình KTĐG đã được GVNVCL áp dụng so vói muc tiêu đào tạo chung của chwơng trình tiếng Anh NVCL

Khi được hỏi về mức độ phù hợp của các loại hình KTĐG đã được GV NVCL áp dụng so với mục tiêu đào tạo chung của chương trình tiếng Anh NVCL, đa số SV được hỏi cho rằng các loại hình KTĐG đang được áp dụng trong chương trình NVCL là phù hợp và rất phù hợp. Tỉ lệ cho rằng các loại hình KTĐG không phù hợp tương đối thấp đến rất thấp (20\% đến $0 \%$ ). Bài tập dự án và kiểm tra giữa kỳ được cho là không phù hợp bởi một tỉ lệ SV cao hơn so với các loại hình KTĐG khác (20\% và 19\%).

Kiểm tra định kỳ, thuyết trình trên lớp, tập bài viết, và bài tập về nhà là các loại hình được đại đa số $\mathrm{SV}$ cho là phù hợp và rất phù hợp (97\% đến 100\%).

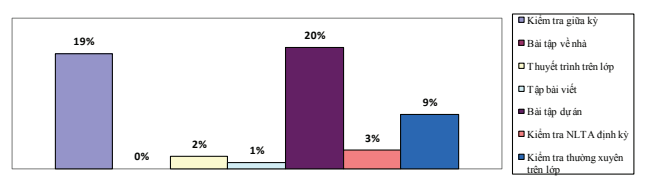

Hình 3. Tỉ lệ $\mathrm{SV}$ cho rằng các loại hình KTĐG đã được GVNVCL áp dụng so với mục tiêu đào tạo chung của chương trình tiếng Anh NVCL là KHÔNG phù hợp

\subsubsection{Tác động của các dạng bài kiểm tra} tới chiến lược và ý thức học tiếng Anh của SV

Khi đề cập đến tác động của các dạng bài kiểm tra đa số $\mathrm{SV}$ được hỏi cho rằng các loại hình KTĐG đang được áp dụng trong chương trình NVCL có nhiều tác động tới chiến lược và ý thức học tiếng Anh của họ. Tỉ lệ cho rằng các loại hình kiểm tra không có tác động tương đối thấp đến rất thấp ( $13 \%$ đến $1 \%)$. Bài tập dự án và kiểm tra giữa kỳ được cho là không có tác động bởi một tỉ lệ SV cao hơn so với các loại hình KTĐG khác (13\%). Các loại hình kiểm tra còn lại được đại đa số SV cho là có tác động ( $94 \%$ đến $99 \%$ ).

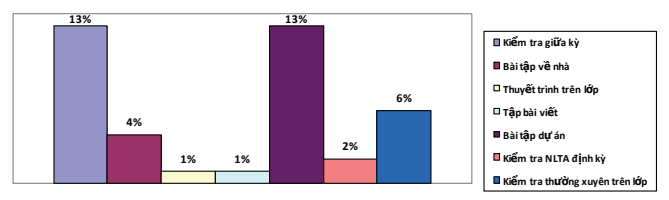

Hình 4. Tỉ lệ SV cho rằng các loại hình KTĐG đã được GVNVCL áp dụng là KHÔNG có tác động tới chiến lược và ý thức học tiếng Anh của SV 
Tuy nhiên tî̉ lệ $\mathrm{SV}$ cho rằng các loại hình kiểm tra đánh giá đang được áp dụng có ít tác động đến chiến lược và ý thức học tiếng Anh của họ cũng là điều đáng suy ngẫm (14\% đến 36\%), trong đó trên dưới $1 / 3$ (31\% đến $36 \%)$ SV cho rằng bài kiểm tra giữa kỳ, bài thuyết trình trên lớp và bài tập dự án ít có tác động đến chiến lược và ý thức của họ. Cần cân nhắc kỹ đến loại hình bài tập dự án và kiểm tra giữa kỳ vì đây là những loại hình được xấp xỉ 50\% (49\% và $44 \%$ ) SV cho là ít có hoặc không có tác động đến chiến lược và ý thức học tiếng Anh của họ.

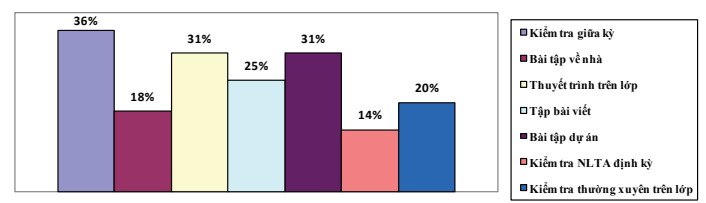

Hình 5. Tỉ lệ $\mathrm{SV}$ cho rằng các loại hình KTĐG đã được GVNVCL áp dụng là ÍT có tác động tới chiến lược và ý thức học tiếng Anh của SV

\subsection{4. Độ tin cậy của các dạng bài kiểm tra}

Về độ tin cậy của các dạng bài kiểm tra, đa số SV ( $62 \%$ đến $83 \%$ ) cho rằng các dạng bài KTĐG nói trên đánh giá đúng NLTA của họ, ngoại trừ bài tập dự án (41\%).

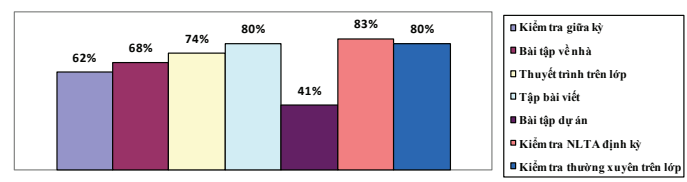

Hình 6. Tỉ lệ SV cho rằng các dạng bài

KTĐG đã được GVNVCL áp dụng là đáng tin cậy

\subsection{Chuoong trình giảng day tiếng Anh NVCL}

Điều tra ý kiến của $\mathrm{SV}$ về chương trình giảng dạy NVCL tập trung vào các vấn đề sau:

- Tính hợp lí của chương trình

- Số giờ dạy của GV nước ngoài/ tổng số giờ

- Sự cần thiết của các nội dung trong phương pháp giảng dạy của $\mathrm{GV}$
- Tỉ lệ phân bố thời lượng giảng bài của GV và thời lượng tham gia hoạt động trên lớp của $\mathrm{SV}$

- Tính phù hợp của các đường hướng giảng dạy

- Tính cần thiết của các yêu cầu trong chương trình môn học

3.3.1. Tính hợp lí của một số yếu tố thuộc chuoong trình tiếng Anh NVCL

Tính hợp lí của chương trình tiếng Anh NVCL được đánh giá dựa trên các yếu tố sau:

- Số tiết học/buổi

- Thời lượng cho 1 học kỳ

- Phân phối nội dung giảng dạy giữa các kỹ năng

- Giáo trình sử dụng

- Mục tiêu chương trình

- Phân phối GV cho các nội dung giảng dạy giữa các kỹ năng

- Phân bổ giờ dạy của GV nước ngoài/ lớp

Đa số $\mathrm{SV}$ (trên 74\%) cho rằng các yếu tố trên của chương trình tiếng Anh NVCL là hợp lí, ngoại trừ việc phân bổ giờ dạy của GV nước ngoài/ lớp. Tỉ lệ cao nhất thuộc về việc phân phối nội dung giảng dạy giữa các kỹ năng (96\%). Tỉ lệ thấp nhất (53\%) thuộc về việc phân bổ giờ dạy của $\mathrm{GV}$ nước ngoài/ lớp. Các yếu tố được trên $90 \% \mathrm{SV}$ cho là hợp lí bao gồm mục tiêu chương trình và số tiết học/buổi.

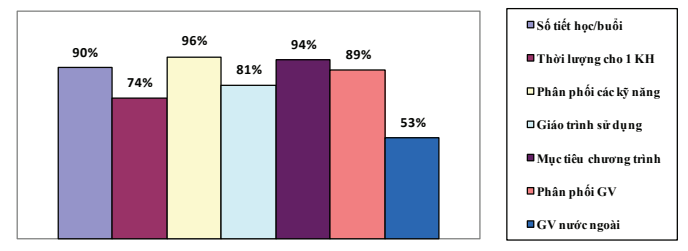

Hình 7 . Tỉ lệ $\mathrm{SV}$ cho rằng các yếu tố nêu trên thuộc chương trình NVCL là hợp lí 
3.3.2. Số giờ dạy của GV nước ngoài/ tổng số giờ

Đa số $\mathrm{SV}$ cho rằng việc $\mathrm{GV}$ nước ngoài giảng dạy trên $50 \%$ số giờ dạy là không hợp lí (91\%). Điều này cho thấy xu hướng muốn được giảng dạy bới cả $G V$ Việt Nam và $G V$ nước ngoài.

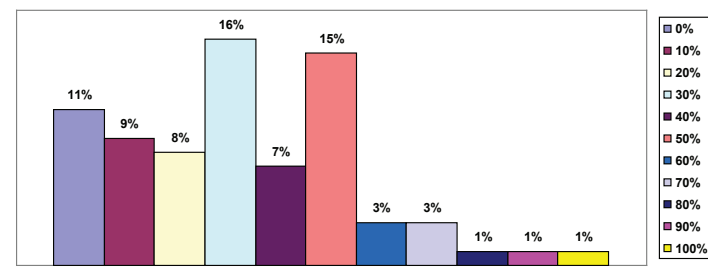

Hình 8. Ý kiến $\mathrm{SV}$ về tỉ lệ giờ giảng của $\mathrm{GV}$ nước ngoài/tổng số giờ giảng

\subsubsection{Sục cần thiết của các đặc điểm liên} quan đến phương pháp giảng dạy của $G V$

Khi được hỏi về sự cần thiết của các đặc điểm liên quan đến phương pháp giảng dạy của $\mathrm{GV}$, đa số $\mathrm{SV}(88 \%$ đến $96 \%)$ cho rằng các đặc điểm sau đây là cần thiết:

- Lấy người học làm trung tâm

- $\quad$ Áp dụng nhiều phương pháp giảng dạy khác nhau cho các mục tiêu và đối trượng giảng dạy khác nhau

- $\quad$ Úng dụng công nghệ thông tin trong giảng dạy

- $\quad$ Áp dụng các phương pháp giảng dạy mới (ví dụ hoạt động theo dự án, giảng dạy theo tiến trình, ...)

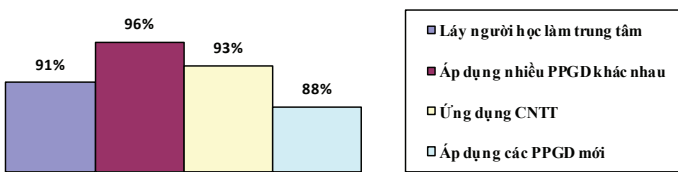

Hình 9. Tỉ lệ SV đồng ý với sự cần thiết của các đặc điểm liên quan đến PPGD của GV

Tỉ lệ SV đồng ý với sự cần thiết của việc áp dụng các PPDG mới trong đó có hoạt động theo dự án tuy tương đối cao nhưng vẫn thấp hơn tỉ lệ ủng hộ với các đặc điểm khác $(88 \%$ so với 91\% đến 96\%). Điều này tương đối nhất quán với tỉ lệ cho rằng $\mathrm{KTĐG} \mathrm{dựa} \mathrm{vào}$ bài tập dự án là không phù hợp $(20 \%$, xem 3.2.2), không hoặc ít có tác động đến ý thức và chiến lược học tập của $\mathrm{SV}$ (13\% đến $31 \%$, xem 3.2.3).

\subsubsection{Tỉ lệ phân bố thời lương giảng bài} của $G V$ và thời lượng tham gia hoạt động trên lóp của $S V$

Tỉ lệ được nhiều SV $(60 \%)$ lựa chọn nhất là GV: $50 \%, \mathrm{SV}: 50 \%$. Điều này cho thấy sinh viên có xu hướng muốn được tự chủ hơn trong lớp học tuy nhiên các em vẫn cần sự hướng dẫn của $\mathrm{GV}$.
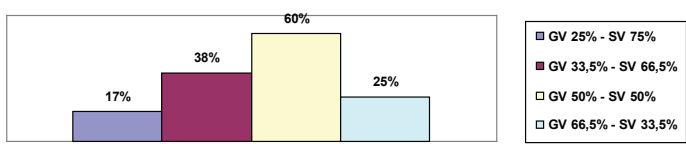

Hình 10. Ý kiến SV về tỉ lệ hợp lí giữa hoạt động của $G V$ và $S V$ trên lớp

\subsubsection{Các đường hướng giảng dạy tiếng} Anh phù hợp với sinh viên NVCL

Các đường hướng giảng dạy tiếng Anh cho sinh viên NVCL sau được khảo sát để tìm ra những đường hướng có tỉ lệ $\mathrm{SV}$ cao cho là phù hợp:

- $\quad$ SV thực hành để phát triển kỹ năng với sự hướng dẫn thực hành của $\mathrm{GV}$

- $\mathrm{GV}$ cung cấp kiến thức nền để $\mathrm{SV}$ áp dụng và thực hành phát triển kỹ năng

- Dạy học thông qua hoạt động và sự tham gia đóng góp của chính người học

- Dạy học dựa trên việc hình thành và phát triển các kỹ năng tự học/tự nghiên cứu của người học

- Dạy học dựa trên sự phân hóa trong môi trường hoạt động học tập tương tác, cộng tác 
- Dạy học dựa trên việc đánh giá, tự đánh giá và cùng đánh giá

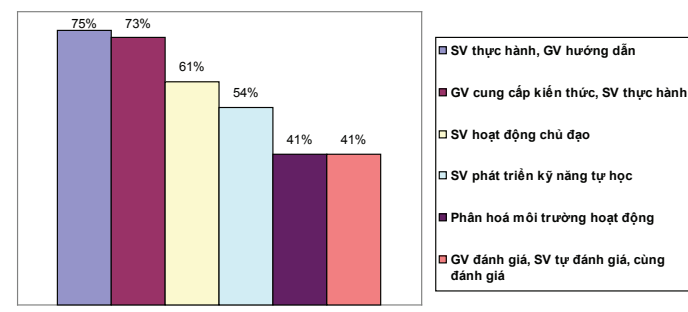

Hình 11. Tỉ lệ SV về ủng hộ các đường hướng giảng dạy của $\mathrm{GV}$

Các đường hướng được nhiều SV lựa chọn hơn là SV thực hành để phát triển kỹ năng với sự hướng dẫn thực hành của $\mathrm{GV}(75 \%)$ và $\mathrm{GV}$ cung cấp kiến thức nền để $\mathrm{SV}$ áp dụng và thực hành phát triển kỹ năng (73\%), kế đến là đường hướng dạy học thông qua hoạt động và sự tham gia đóng góp của chính người học (61\%). Các đường hướng dạy học dựa trên sự phân hóa trong môi trường hoạt động học tập tương tác, cộng tác; dạy học dựa trên việc đánh giá, tự đánh giá và cùng đánh giá không được nhiều $\mathrm{SV}$ cho là phù hợp với tỉ lệ ủng hộ là 41\%.

\subsubsection{Mong muốn của SV về tính rõ ràng} và phù hợp của các yêu cầu trong các học phần thuộc chuoong trình

Điều tra khảo sát mong muốn của $\mathrm{SV}$ NVCL về tính rõ ràng và phù hợp của các yêu cầu trong các học phần thuộc chương trình tập trung vào các tiêu chí sau:

- Đường hướng và nguyên tắc giảng dạy của học phần phù hợp với mục tiêu chung của chương trình NVCL

- Các hoạt động của SV được nêu rõ trong nội dung từng học phần

- Các hoạt động của GV được nêu rõ trong nội dung từng học phần

- Các nội dung KTĐG được nêu chi tiết trong nội dung từng học phần
- Giáo trình và tài liệu bổ trợ được nêu chi tiết trong nội dung từng học phần

- Học phần có mục tiêu rõ ràng và phù hợp với mục tiêu chung của chương trình

Đa số SV (tối thiểu là $72 \%$ ) cho rằng các yêu cầu trong các học phần phải rõ ràng và phù hợp với mục tiêu chung của chương trình trong đó tiêu chí đường hướng và nguyên tắc giảng dạy của học phần phải phù hợp với mục tiêu chung của chương trình NVCL được nhiều SV tán thành nhất (93\%). Các tiêu chí còn lại đều được đa số SV (từ 72\% đến 91\%) cho là cần thiết.

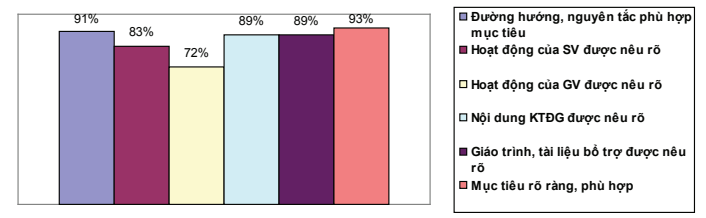

Hình 12. Tỉ lệ SV mong muốn về tính rõ ràng và phù hợp trong các yêu cầu của chương trình

\section{4. Đội ngũ giáo viên giảng dạy}

Việc khảo sát đánh giá của $\mathrm{SV}$ về $\mathrm{GV}$ tham gia giảng dạy chương trình tập trung vào các tiêu chí sau:

- $\quad$ GV giảng dạy nhiệt tình trong lớp học

- GV truyền đạt kiến thức tốt

- $\quad \mathrm{GV}$ chuẩn bị bài cẩn thận

- $\mathrm{GV}$ mở rộng kiến thức cho $\mathrm{SV}$

- $\quad$ Giáo viên áp dụng PPGD phù hợp

- GV thông báo cho $\mathrm{SV}$ về chương trình giảng dạy mỗi học phần từ đầu học kỳ

- $\quad \mathrm{GV}$ quan tâm từng $\mathrm{SV}$ trong quá trình giảng dạy

- GV sử dụng nhiều tài liệu bổ trợ trong quá trình giảng dạy

- GV thực hiện đúng theo chương trình học phần thông báo cho SV từ đầu mỗi học kỳ 
Đa số $\mathrm{SV}$ đánh giá cao về đội ngũ $\mathrm{GV}$, trong đó $99 \% \mathrm{SV}$ cho rằng $\mathrm{GV}$ nhiệt tình trong giảng dạy, có kĩ năng và phương pháp truyền đạt kiến thức tốt và chuẩn bị bài cẩn thận trước khi đến lớp (88\%). Ngoài ra, khi giảng dạy trên lớp, $\mathrm{GV}$ cũng thường xuyên mở rộng kiến thức và được $\mathrm{SV}$ đánh giá cao (83\%). Đa số $\mathrm{SV}$ cho rằng $\mathrm{GV}$ có phương pháp giảng dạy dễ hiểu và phù hợp với trình độ của $\mathrm{SV}(77 \%)$. Điều này cho thấy đội ngũ cán bộ giảng dạy là một điểm mạnh của chương trình.
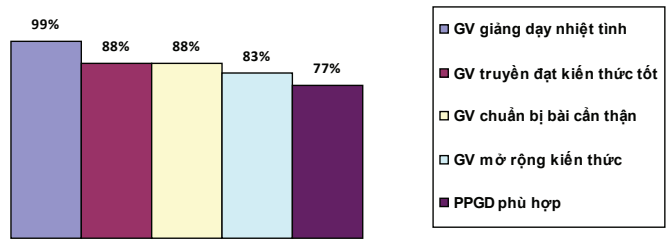

Hình 13a. Tỉ lệ SV đánh giá GV theo các tiêu chí nêu ra ở mức tốt và xuất sắc

Đa số SV đánh giá cao về các hoạt động của GV. 93\% SV xác nhận về việc GV có thông báo cho $\mathrm{SV}$ về chương trình giảng dạy mỗi môn học từ đầu học kỳ, GV quan tâm từng $\mathrm{SV}$ trong quá trình giảng dạy, sử dụng nhiều tài liệu bổ trợ trong quá trình giảng dạy và thực hiện đúng theo chương trình môn học thông báo cho SV từ đầu mỗi học kỳ với tỷ lệ lần lượt $78 \%, 72 \%$, và $67 \%$.

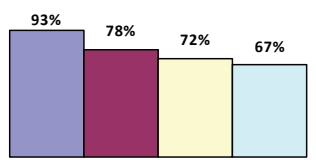

口Thông báo cho SV về C TGD - $\mathrm{Q}$ uan tâm đến từng $\mathrm{SV}$ ๑Sử dụng n hiều tài liệu bổ trọ ๑Thực hiện đúng chương trình

Hình 13b. Tỉ lệ SV đánh giá GV theo các tiêu chí nêu ra ở mức tốt và xuất sắc

\subsection{Hoạt động ngoại khóa của sinh viên}

Việc khảo sát quan điểm của SVNVCL về sự cần thiết của các hoạt động ngoại khóa đối với quá trình học tiếng Anh của họ tập trung vào những hoạt động sau:
- Tổ chức và/hoặc tham gia CLB nói tiếng Anh

- $\quad$ Đi tham quan các bảo tàng, di tích lịch sử, văn hóa

- Tham gia các kỳ thi Olympic tiếng Anh

- $\quad$ Tổ chức các buổi Gala SV NVCL

- Giao lưu với SV các cơ sở/trung tâm giảng dạy tiếng Anh tại Hà Nội

- Giao lưu với SV các trường đại học trong nước và quốc tế

- Hướng dẫn các lưu học sinh nước ngoài tại Trường Đại học Ngoại ngữ trong cuộc sống và học tập

Phần lớn các SV đều thấy sự cần thiết của các hoạt động ngoại khóa nêu ra đối với quá trình học tiếng Anh của SV NVCL. Chiếm tỷ lệ cao nhất trong các hoạt động này là hoạt động giao lưu với $\mathrm{SV}$ các trường đại học trong nước và quốc tế $(90 \%) .86 \% \mathrm{SV}$ được hỏi co rằng việc tham gia hướng dẫn các lưu học sinh nước ngoài tại Trường Đại học Ngoại ngữ trong cuộc sống và học tập là cần thiết cho quá trình học tập tiếng Anh của họ. Các hoạt động khác được SV đánh giá cao về mức độ cần thiết bao gồm tổ chức các buổi Gala SVNVCL (82\%), giao lưu với SV các cơ sở/trung tâm giảng dạy tiếng Anh tại Hà Nội $(82 \%)$ và tổ chức, tham gia CLB nói tiếng Anh 80\%). Tỉ lệ ủng hộ việc đi tham quan các bảo tàng, di tích lịch sử, văn hóa chiếm 71\%. Tuy nhiên, tỷ lệ sinh viên ủng hộ việc tham gia các kỳ thi Olympic tiếng Anh chưa cao, chỉ ở mức 57\%.

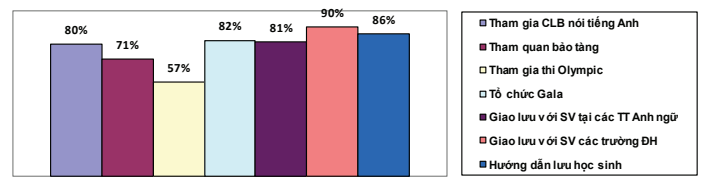

Hình 14. Tỉ lệ SV ủng hộ về sự cần thiết của các hoạt động ngoại khóa 
3.6. Chiến lược và phuơng pháp hoc tập của $S V$

Quan điểm của SVNVCL về tính cần thiết của các hoạt động liên quan đến chiến lược và phương pháp học tập xoay quanh các vấn đề sau:

- Xây dựng chiến lược học tiếng Anh từ học kỳ đầu tham gia chương trình tiếng Anh NVCL

- Học tiếng Anh ngoài lớp học theo cặp, nhóm

- Học tiếng Anh ngoài lớp học theo dự án

- Tự học, tự nghiên cứu

- Tham quan thực tế, thực hành

- Học tiếng Anh bằng cách tham khảo, tư vấn người khác

Hầu hết SV $(99 \%)$ cho rằng việc xây dựng chiến lược và phương pháp học tập từ học kỳ đầu tham gia chương trình tiếng Anh NVCL là cần thiết. Các hoạt động tự học/tự nghiên cứu, tham quan thực tế/thực hành, học tiếng Anh ngoài lớp học theo cặp, nhóm và học tiếng Anh bằng cách tham khảo, tư vấn người khác chiếm tỉ lệ cao và khá cao lần lượt là $94 \%, 92 \%, 88 \%$ và $86 \%$. Hoạt động học tiếng Anh ngoài lớp học theo dự án được ít SV cho là cần thiết hơn cả so với các hoạt động khác với tỷ lệ ủng hộ là $63 \%$.

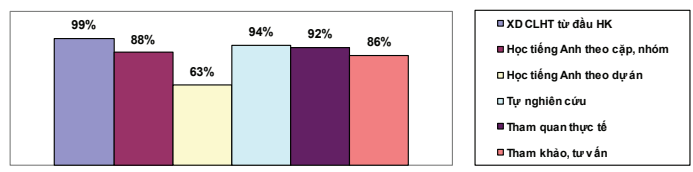

Hình 15. Tỉ lệ SV ủng hộ tính cần thiết của các hoạt động liên quan đến chiến lược và phương pháp học tập

\subsection{Hoạt động hỗ trọ̣ của nhà trường}

Quan điểm của SV về sự cần thiết của việc hỗ trợ của Trường ĐHNN-ĐHQGHN cho quá trình học tập của họ dựa vào các yếu tô và các hoạt động sau:

- Phòng học được trang bị các thiết bị nghe nhìn hỗ trợ quá trình học tiếng

- Định hướng và hướng dẫn đầu năm học cho SV

- Các môn chung và chuyên ngành được bố trí hợp lý, không làm ảnh hưởng tới việc học tiếng Anh của SV

- $\quad$ SV được thông báo về các chủ trương, chính sách kịp thời và nhanh chóng

- Hệ thống thư viện của nhà trường có đủ các sách, giáo trình, tài liệu tham khảo phục vụ việc học tiếng Anh cho SV NVCL

- Hệ thống phòng đọc của nhà trường phù hợp với nhu cầu của SV NVCL

- Chương trình cố vấn học tập của nhà trường được triển khai trong chương trình tiếng Anh NVCL

- Các đơn vị trong nhà trường liên lạc, phối hợp với nhau trong hoạt động quản lý SV

Hầu hết SV (từ 91\% trở lên) đánh giá cao sự cần thiết của các hoạt động hỗ trợ của Trường ĐHNN-ĐHQGHN trong quá trình học tập, trong đó $98 \%$ cho rằng việc bố trí hợp lí các môn chung và chuyên ngành và nhận được thông báo về chủ trương, chính sách là cần thiết. Các hoạt động và yếu tố khác đều nhận được sự ủng hộ cao về tính cần thiết gồm phòng học được trang bị các thiết bị nghe nhìn hỗ trợ quá trình học tiếng $(97 \%)$, thư viện cung cấp đầy đủ sách, giáo trình, tài liệu tham khảo $(95 \%)$, Nhà trường, GV định hướng và hướng dẫn đầu năm học cho $\mathrm{SV}$, hệ thống phòng đọc của Nhà trường phù hợp với nhu cầu của $\mathrm{SV}$, các đơn vị trong nhà trường liên lạc, phối hợp với nhau trong hoạt động quản lý 
SV $(94 \%)$, chương trình cố vấn học tập của nhà trường được triển khai trong chương trình tiếng Anh NVCL (91\%).
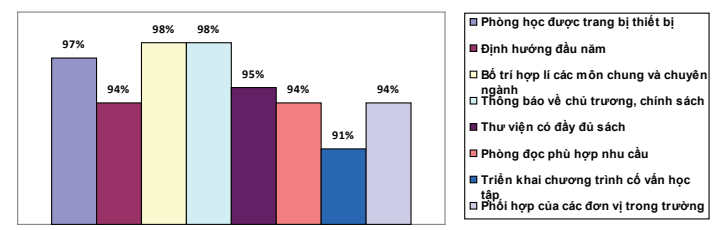

Hình 16. Tỉ lệ SV đồng ý với tính cần thiết của các hoạt động hỗ trợ của Nhà trường

\section{Kết luận}

Qua khảo sát quan điểm, ý kiến của $\mathrm{SV}$ tham gia chương trình đào tạo tập trung tiếng Anh NVCL về thực trạng đào tạo của chương trình, chúng tôi thấy đây là một hình thức đào tạo phù hợp với đường hướng giáo dục dựa vào chuẩn đầu ra. Nhìn chung, $\mathrm{SV}$ đánh giá cao các yếu tố liên quan đến các hoạt động dạy và học của chương trình. Những điểm mạnh nổi bật của chương trình là đội ngũ cán bộ giảng dạy nhiệt tình, chuyên nghiệp; chương trình được thiết kế hợp lí, phù hợp với mục tiêu và chuẩn đầu ra đề ra với các hoạt động hỗ trợ người học nội khóa và ngoại khóa phong phú, có hiệu quả, đường hướng, phương pháp giảng dạy, giáo trình, tài liệu sử dụng đa dạng, được điểu chỉnh cho phù hợp với $\mathrm{SV}$ thuộc nhiều trình độ khác nhau, các hình thức KTĐG phù hợp với mục tiêu của chương trình và có độ tin cậy cao. Kết quả khảo sát còn cho thấy SV NVCL rất quan tâm đến những phương pháp chiến lược học tập của cá nhân và luôn trăn trở tìm tòi những phương cách làm nâng cao năng lực tiếng Anh của mình. Điều này cho thấy tác động tích cực của chương trình đối với động cơ và ý thức học tập của SV. Đây là một hình thức đào tạo nên được duy trì, phát huy và nhân rộng nhằm tạo ra nguồn nhân lực chất lượng cao phục vụ quá trình hội nhập và toàn cầu hoá ngày một sâu rộng của nước ta.

\section{Tài liệu tham khảo}

Berlach, R. G. (2004). Outcomes-based education and the death of knowledge. Paper presented at the Australian Association for Research in Education, The University of Melbourne.

Cawelti, G. (1994). High school restructuring: A national study. Arlington, V. A.: Educational Research Service.

Clark, E. \& Clark, P. (2000). Taking the educational show on the road: The promises and pitfalls of intensive teaching in offshore postgraduate coursework programs. International Education, 4(1).

Damer, T. E. (2008). Attacking Faulty Reasoning: A Practical Guide to Fallacy-free Arguments Cengage Learning.

Davies, W. M. (2006). Intensive Teaching Formats: A review. Issues in Educational Research, 16(1).

Evans, K. (1994). Research on OBE: What we know and don't know. Educational Leadership, 51(6), 79-82.

Finger, G. \& Penney, A. (2001). Investigating modes of delivery in teacher education: A review of modes of delivery. Paper presented at the Annual Conference of the Australian Association for Research in Education, Fremantle, Western Australia.

Gaubatz, N. (2003). Course scheduling formats and their impact on student learning. The National Teaching and Learning Forum, 12(1).

Gose, B. (1995). One-course-at-a-time 'block plan' re-examined by college that adopted it 25 years ago. The Chronicle of Higher Education, 41(47), A28.

Grant, D. B. (2001). Using block courses for teaching logistics. International Journal of Physical Distribution and Logistics Management, 31(7/8), 574-584. 
Henebry, K. (1997). The impact of class schedule on student performance in a financial management course. Journal of Education for Business, 73(2), 114-120.

Jonas, P. M., Weimer, D. \& Herzer, K. (2004). Comparison of traditional and nontraditional undergraduate business degree programs - adult education. Journal of Instructional Psychology, 28(3), 161-168.

Johnson, D. M. (1992). Approaches to Research in Second Language Learning. Longman.

Killen, R. (2000). Teaching strategies for outcomes-based education. Cape Town: Juta Educational Publishers.

Kohn, A. (1993). Turning learning into a business: Concerns about total quality. Educational Leadership, 51(1), 58-61.

Mackey, A., and Gass, S.M. (2005). Second Language Research: Methodology and Design. Lawrence Erlbaum Associates Publishers.

Nunan, D. (1992). Research Methods in Language
Learning. CUP.

Petrowsky, M. C. (1996). The two-week summer microeconomics course: Success or failure? ERIC Document Reproduction Service, ED 396779 .

Scott, P. A. \& Conrad, C. F. (1992). A critique of intensive courses and an agenda for research. Higher Education: Handbook of Theory and Research, 8(411-459).

Spady, W. G. (1994). Outcome-based education: Critical issues and answers. Arlington: American Association of School Administrators.

van Scyoc, L. \& Gleason, J. (1993). Traditional or intensive course lengths? A comparison of outcomes in Economics learning. Journal of Economics Education, 24, 15-22.

Wlodkowski, R. J. (2003). Accelerated learning in colleges and universities. New Directions for Adult and Continuing Education, 97(Spring), 5-15.

\title{
STUDENTS' VIEWS ON THE CURRENT SITUATION OF THE VNU INTERNATIONAL STANDARD ENGLISH TEACHING PROGRAMME
}

\author{
Huynh Anh Tuan ${ }^{1}$, Do Thi Anh Thu²
}

${ }^{1}$ Faculty of Post-Graduate Studies, VNU University of Languages and International Studies, Pham Van Dong, Cau Giay, Hanoi, Vietnam

${ }^{2}$ Faculty of Foreign Languages, Vietnam Maritime University, 484 Lach Tray, Ngo Quyen, Hai Phong, Vietnam

$\mathrm{PhD}$. Research Student, VNU University of Languages and International Studies

\begin{abstract}
This paper reflects and analyzes the current situation of teaching English to International Standard Programme (ISP) students based on the survey results of the students' opinions of the programme's teaching and learning activities to make some suggestions for the improvement of the programme and students' outcome quality. The survey results indicated that despite some issues yet to be resolved and improved, most of the students claimed that the programme was significantly effective and suitable for outcome-based education.
\end{abstract}

Keywords: International Standard Programme (ISP), intensive English teaching model, outcomebased education, English proficiency 